Title:

Author(s):
Structure versus Properties in High-Temperature Superconductors

\section{RECEIVED SEP 231996}

P. Chris

Marilyn Hawley, MST-CMS

Markku Salkola, T-11/VIS

Joe Thompson, MST-10

Stuart Trugman, T-11

Yohsuke Yoshinari, MST-10

DOE Office of Scientific and Technical Information (OSTI)

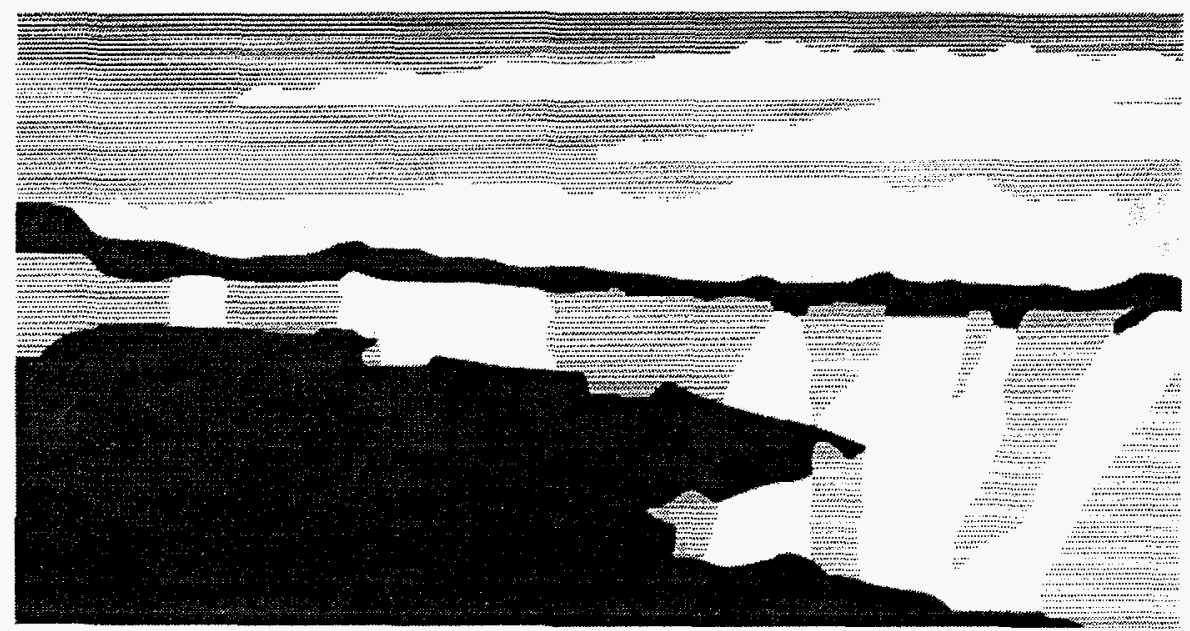

Los Alamos National Laboratory, an affirmative action/equal opportunity employer, is operaled by the University of California for the U.S. Department of Energy under contract W-7405-ENG-36. By acceptance of this article, the publisher recognizes that the U.S. Government retains a nonexclusive, royaltyfree license to publish or reproduce the published form of this contribution, or to allow others to do so, for U.S. Government purposes. The Los Alamos National Laboratory requests that the publisher identify this article as work performed under the auspices of the U.S. Department of Energy. 


\section{DISCLAIMER}

Portions of this document may be illegible in electronic image products. Images are produced from the best available original document. 


\section{DISCLAIMER}

This report was prepared as an account of work sponsored by an agency of the United States Government. Neither the United States Government nor any agency thereof, nor any of their employees, makes any warranty, express or implied, or assumes any legal liability or responsibility for the accuracy, completeness, or usefulness of any information, apparatus, product, or process disclosed, or represents that its use would not infringe privately owned rights. Reference herein to any specific commercial product, process, or service by trade name, trademark, manufacturer, or otherwise does not necessarily constitute or imply its endorsement, recommendation, or favoring by the United States Government or any agency thereof. The views and opinions of authors expressed herein do not necessarily state or reflect those of the United States Government or any agency thereof. 


\title{
Structure versus Properties in High-Temperature Superconductors
}

James L. Smith*, F. Chou (Ames Laboratory, University of Iowa), Zachary Fisk (National High Magnetic Field Laboratory, Florida State University), Stephen Foltyn,

P. Chris Hammel, Marilyn Hawley, D. Johnson (Ames Laboratory, University of Iowa), Joseph Martindale, Evangelina Moshopolou, John Neumeier, Markku Salkola, Brian Statt (University of Toronto), Joe Thompson, Stuart Trugman, and Yohsuke Yoshinari

\begin{abstract}
This is the final report of a one-year, Laboratory-Directed Research and Development (LDRD) project at the Los Alamos National Laboratory (LANL). High-temperature superconductors (HTS) have crystal structures that make them a distorted subset of Perovskite materials. The complexity of the superconductors arising from the large number of elements that they possess gives us all sorts of imperfect regions in our materials, and these regions tend to show Perovskite-like properties. This project sought to expand our fundamental understanding of high-temperature superconductors by combining experimental and theoretical studies of this class of novel materials. The research objectives were to interrelate structural and superconducting properties of HTS materials to further understand parameters influencing their behavior in important power and electronic applications.
\end{abstract}

\section{Background and Research Objectives}

HTS have crystal structures that make them a distorted subset of Perovskite materials. The complexity of the superconductors arising from the large number of elements that they possess gives us all sorts of imperfect regions in our materials and these regions tend to show Perovskite-like properties. This is the source of the daunting materials problems, which we have seen and only now are we solving, and presents us with an opportunity to explore the use of these mixed-up materials. Indeed, they appear to be useful for such things as magneticstorage readers and thin-film capacitors. Great progress has been made in understanding both

* Principal investigator, e-mail: smith_james_1@lanl.gov 
fundamental and applied issues for HTS over the last eight years. We must probe the "mesoscale" relationship between structure and properties, which includes such details as screw dislocations, vortex structure, structural instabilities (driven by charge and spin polarization), and surface chemistry and physics. We must also study related materials to help understand the superconductors, some of which may have technological uses in their own rights. This project sought to expand our fundamental understanding of HTS by combining experimental and theoretical studies of this class of novel materials. The research objectives entailed interrelating structural and superconducting properties of HTS materials to further understand parameters influencing their behavior in important power and electronic applications.

\section{Importance to LANL's Science and Technology Base and National R\&D Needs}

This project supports Los Alamos core competencies in nuclear and advanced materials as well as complex experimentation and measurement. HTS have numerous applications both in improving electrical power efficiency as well as in developing the next generation of advanced electronic devices. Many of these uses have commercial as well as defense benefits. It not only affords Los Alamos with international recognition, but serves as a fundamental underpinning for understanding and improving HTS materials properties for applications.

\section{Scientific Approach and Results to Date}

The temperature dependence of the anisotropic planar oxygen nuclear spin-lattice relaxation rate has been measured for both the normal and superconducting states of an aligned powder sample of $\mathrm{YBa}_{2} \mathrm{Cu}_{3} \mathrm{O}_{6.96}$ enriched with ${ }^{17} \mathrm{O}$. Measurements of the relaxation rates with the magnetic field $\mathrm{H}_{\mathrm{O}}$ perpendicular to the $\mathrm{c}$ axis were made possible with a new nuclear magnetic resonance (NMR) technique to overcome the random alignment of the powder in the ab plane. The data for the normal state show that ${ }^{17} \mathrm{~W}_{1 \|}$ (the relaxation rate measured with $\mathrm{H}_{0}$ in the ab plane and parallel to the copper-oxygen bond axis) has a different temperature dependence than ${ }^{17} \mathrm{~W}_{1} \perp\left(\mathrm{H}_{\mathrm{O}}\right.$ in the ab plane and perpendicular to the copper-oxygen bond axis) and ${ }^{17} \mathrm{~W}_{1 \mathrm{c}}\left(\mathrm{H}_{\mathrm{O}}\right.$ parallel to the $\mathrm{c}$ axis) below about $150 \mathrm{~K}$. This difference ceases at $\mathrm{Tc}$, with all three rates having the same temperature dependence in the superconducting state. The deviation of ${ }^{17} \mathrm{~W}_{1 \|}$ from ${ }^{17} \mathrm{~W}_{1 \perp}$ and ${ }^{17} \mathrm{~W}_{1 \mathrm{c}}$ occurs at approximately the same temperature as the opening of the so-called "spin gap" as determined by Knight shift data, suggesting some connection between these phenomena. The different temperature dependencies for the normal- 
state data are inconsistent with the currently accepted model of the NMR data in the cuprates, which holds that only a single spin degree of freedom exists in the $\mathrm{CuO}_{2}$ planes. Calculations of the oxygen relaxation rates for a single-spin susceptibility that included a q-independent hyperfine coupling in addition to the standard-model-transferred hyperfine coupling were unable to account for the experimental results. Therefore, a second spin degree of freedom in the $\mathrm{CuO}_{2}$ planes must exist.

These results support a model proposed by T. Egami, et al. in which he proposed that local deviations from the average crystal structure observed in neutron-scattering measurements shift the energy of oxygen orbitals. This energy shift results in the appearance of a second rather flat band at the Fermi surface. The behavior of this second band is consistent with the temperature-dependent anisotropy we have observed.

Our study of the relationship between structural modulations and phase separation in oxygen-doped $\mathrm{La}_{2} \mathrm{CuO}_{4}+\mathrm{d}$ has led us to two conclusions. Our results in conjunction with those of neutron scattering and La NMR lead us to conclude that oxygen ordering occurs on a microscopic scale in this compound. The ordered interstitial oxygen gives rise to a distribution of tilts of the $\mathrm{CuO}_{6}$ octahedra. A sawtooth modulation of the octahedral tilt, in which the sign of the tilt changes when the tilt reaches a maximum value provides a location for the interstitial oxygen. This leads to stripe ordering of interstitial oxygen. In addition, we show that macroscopic phase separation is correlated with the onset of the orthorhombic structural phase transition because oxygen mobility is inhibited in the tetragonal phase where the $\mathrm{CuO}_{6}$ octahedra are not tilted.

We have used a combination of analytical and numerical many-body techniques to study several important aspects of HTS controlled by the simultaneous presence of electron-electron, electron-lattice, and multiband coupling.

We have used exact diagonalization numerical techniques to study $\mathrm{Cu}-\mathrm{O}$ chains and planes in multiband models and shown how the competition between the difference in orbital energy levels and the Hubbard electron-electron interaction controls $\mathrm{Cu}-\mathrm{O}$ charge-transfer instabilities between Mott and band states. We also find that lattice deformations enhance charge-transfer fluctuations around these instabilities. Thus, electron-electron interactions strongly enhance specific lattice deformation couplings and may control competition between (anti)ferroelectricity and superconductivity. We are collaborating with Egami, et al. (University of Pennsylvania) to interpret inelastic neutron-scattering evidence for the lattice deformation and phonon softening and its relationship to spin pseudogaps opening in HTS compounds.

In a related study, we have used renormalization-group analysis to demonstrate that electron-lattice coupling in a multiband extended Peierls-Hubbard $\mathrm{Cu}-\mathrm{O}$ model can (at least in 
one dimension) favor superconductivity over spin- or charge-density-wave instabilities. We have studied the regime of superconducting instability as a function of electron-electron and electron-lattice coupling and hole doping density. Importantly, the superconducting regime is strongly enhanced by $\mathrm{Cu}-\mathrm{O}$ hybridization and next-neighbor Hubbard interactions, even for weak electron-lattice coupling.

Since the evidence for effective electron-lattice coupling is now clear, our earlier studies of "spin-charge-lattice" deformation "polarons," describing specific deformations around holes, becomes especially important for understanding differences between "local" and "global" structure in complex electronic materials. For example, recent inelastic neutron scattering clearly supports charge-ordered (striped) superlattice phases at commensurate hole density. It is critical that polaron signatures are fully appreciated, so that correlations of optical, magnetic, and structural experimental probes are accurately interpreted in complex oxides. We have therefore completed a detailed numerical and analytical study of a three-site $\mathrm{Cu}-\mathrm{O}$ model for polaron tunneling, including complete nonlinear and nonadiabatic effects. We have carefully predicted experimental signatures and compared them with structural (Los Alamos neutron PDF and XAFS) and optical (infrared, Raman) data. The agreement with various "anomalous" features in available data is encouraging and points to the importance of directional polarizability (quantum paraelectric) fluctuations in layered superconducting oxides.

We have also worked closely with Migliori and collaborators to understand the anomalous resonant ultrasound measurements on $\mathrm{La}_{(2-x)} \mathrm{Sr}_{\mathbf{X}} \mathrm{CuO}_{4}$ in terms of ordering and critical fluctuations of the tilting and scissoring modes of the $\mathrm{CuO}_{6}$ octahedra.

There is interesting recent evidence for $\mathrm{d}$-wave pairing in high-temperature superconducting oxides from Josephson tunneling and other experiments. We have therefore considered the effects of impurities in a d-wave BCS superconductor and shown that a single impurity produces a quasi-bound state. The geometry of these impurity-induced states is dictated by the anisotropy of the $d$-wave gap. These states are highly anisotropic and have long-range tails along the directions of vanishing gap. They differ in important respects from the midgap states in s-wave superconductors. We have discussed the relevance of these states to the recent NMR experiments on the Zn-doped cuprates, which show substantial local variations in the NMR signal for sites close to the impurities. These long-range tails lead to large overlaps between impurity states and to delocalization in the impurity band in d-wave superconductors. We are developing a nonlinear sigma model description of the localizationdelocalization transition for the long-range hopping impurity band.

This project has combined experimental NMR and resonant ultrasound measurements with interpretations derived from theoretical models to relate structural and superconducting properties of selected HTS compounds successfully. 


\section{Publications}

A.V. Balatsky, A. Rosengren, and B.L. Altshuler, "Impurities and Quasi-One Dimensional Transport in a D-Wave Superconductor," Phys.Rev. Lett., 73, 720 (1994).

J. Bonca and A.V. Balatsky, "Composite Operators for BCS Pairing," ZhETP Letters, 59, 202 (1994).

A.V. Balatsky, "Impurities and Conductivity in a D Wave Superconductor," in Strongly Correlated Electronic Materials, K. Bedell, et al., editors, Addison-Wesley (1994).

A.V. Balatsky, D. Pines, and P. Monthoux, "The Influence of Imperfections on the Low Temperature Properties of a D-Wave Superconductor," Phys.Rev.B, 50582 (1994).

M.I. Salkola, A.R. Bishop, S.A. Trugman, and J. Mustre de Leon, "Correlation-Function Analysis of Coupled Electron-Phono Systems: Signatures of Polaron Tunneling," in Anharomic Properties of High $T_{c}$ Cuprates, World Scientific, (1994).

A.R. Bishop, "Lattice and Multiband Electronic Effects in HTS Superconductors," in Strongly Correlated Electronic Materials, K. Bedell, et al., editors, Addison-Wesley, (1994).

M.I. Salkola, A.R. Bishop, J. Mustre de Leon, and S.A. Trugman, "Dynmaic Polaron Tunneling in $\mathrm{YBa}_{2} \mathrm{Cu}_{3} \mathrm{O}_{7}$ : Optical Response and Inelastic Neutron Scattering," Phys.Rev. $B(R C), 49,3671$ (1994).

M.I. Salkola and A.V. Balatsky, "Superconducting Fluctuations in Multi-band ID Hubbard Model," Phys.Rev. B, 51, 15267 (1995).

A.V. Balatsky, M. I. Salkola, and A. Rosengren, "Impurity Induced Bound States in D-Wave Superconductor," Phys.Rev. B, 51, 15547 (1995).

E. Abrhams, A.V. Balatsky, D.J. Scalapino, and J.R. Schrieffer, "Properties of Odd Gap Superconductors," Phys.Rev. B, 52, 1271 (1995).

A.V. Batatsky, and M. Salkola, "Impurity Induced States and Absence of Quasiparticle Localization in Disordered d-Wave Superconductors," Phys.Rev.Lett., in press, (1995).

J. Mustre de Leon, I. Baristic, A.R. Bishop, S.D. Conradson, and S.A. Trugman, reply to comment on "Polaron Origin for Anharmonicity of the Axial Oxygen in $\mathrm{YBa}_{2} \mathrm{Cu}_{3} \mathrm{O}_{7}$," Phys.Rev.Lett., 75, 584, (1995).

A. Bussmann-Holder and A.R. Bishop, "Anharmonicity in the c-Direction of High-Tc Oxides," Phys.Rev.B, 51, 6640 (1995).

S. Matveenko, A.R. Bishop, and A. Balatsky, "Superconductivity Fluctuations in a 1Dimensional, 2-Band Electron-Phonon Model with Strong Electron-Electron Repulsion," Phys.Rev.B , submitted, (1995).

A.R. Salkola, A.R. Bishop, S.A. Trugman, and J. Mustre de Leon, "Correlation-Function Analysis of Nonadiabatic and Nonlinear Systems: Polaron Tunneling," Phys. Rev. B, 51, 8878 (1995). 
A.R. Bishop, "Local Polarizability, Structure, and Charge-Trasnfer in HTC Superconductors," J. Superconductivity, in press, (1995).

P.C. Hammel, B.W. Statt, R.L. Martin, S.-W. Cheong, F.C. Chou, and D.C. Johnston, "Localized Holes in Superconducting Lanthanum Cuprate," Phys. Rev. Lett., submitted, (1995).

B.W. Statt, P.C. Hammel, Z. Fisk, S.-W. Cheong, F.C. Chou, and D.C. Johnston, "Oxygen Ordering and Phase Separation in La2CuO4+delta," Phys Rev. B, in press, (1995).

J.A. Martindale, P.C. Hammel, W.L. Hults, and J.L. Smith, "Temperature Dependence of the Anisotropic Planar Oxygen Nuclear Spin-Lattice Relaxation Rate in YBa2Cu3O6.96," Phys.

Rev. Lett., (1995). 